\title{
Autorrelato de diabetes e atividade física no Brasil
}

\section{Self-reported of diabetes and physical activity in Brazil}

\author{
Thamires Lorenzet Cunha Seus ${ }^{1}$ \\ Fernando Vinholes Siqueira ${ }^{1,5}$ \\ Denise Silva da Silveira ${ }^{2}$ \\ Elaine Tomasi ${ }^{2}$ \\ Elaine Thumé ${ }^{3}$ \\ Suele Manjourany Silva ${ }^{4}$ \\ Alitéia Dilelio ${ }^{4}$ \\ Roberto Xavier Piccini ${ }^{2}$ \\ Luiz Augusto Facchini ${ }^{2}$
}

\section{Resumo}

Embora exista um consenso de que a prática de atividade física (AF) ajuda a prevenir o Diabetes Mellitus (DM) e melhorar a qualidade de vida destes indivíduos, ainda é alta a prevalência de inatividade física entre os diferentes grupos etários de pessoas. $\mathrm{O}$ presente artigo descreve a prevalência de autorrelato de DM, o nível de AF desta população e variáveis associadas a este desfecho em adultos e idosos brasileiros. Foi realizado um estudo transversal de base populacional com amostra de 12.402 adultos e 6.624 idosos de 23 estados brasileiros. A prevalência de autorrelato de DM na população de adultos foi de 3,5\% (IC95\% 3,2-3,9), enquanto nos idosos foi de 16,9\% (IC95\% 15,9-17,8). Em relação à prática de AF observou-se que entre os indivíduos que relataram ter DM, 82,6\% (IC95\% 79,0-86,1) dos adultos e 88,2\% (IC95\% 86,3-90,1) dos idosos foram considerados insuficientemente ativos. Por outro lado, entre os indivíduos que não relataram DM a prevalência de indivíduos insuficientemente ativos entre os adultos e idosos foi de $82,5 \%$ (IC95\% 81,9-83,2) e 86,3\% (IC95\% 85,4-87,2) respectivamente. É a alta prevalência de inatividade física entre os indivíduos que relatam ou não o DM.

\section{Palavras-chave}

Diabetes, Atividade Motora, Epidemiologia.

\begin{abstract}
Although there is a consensus that the practice of physical activity (PA) helps prevent Diabetes Mellitus (DM) and improve the quality of life of individuals, it is still a high prevalence of physical inactivity among different age groups of people. This article describes the prevalence of self-report of DM, the level of $P A$ of this population and variables associated with this outcome in Brazilian adults and elderly individuals. A cross-sectional population-based was conducted with a sample of 12,402 adults and 6,624 elderly individuals from 23 Brazilian states. The prevalence of self-reported DM in the adult population was 3.5\% (IC95\% 3,2-3,9), while in the elderly one was 16.9\% (IC95\% 15,9-17,8). Regarding the PA practice, it was observed that among individuals reporting diabetes, $82.6 \%$ (95\% CI 79.0-86.1) of adults and 88.2\% (95\% CI 86.3-90.1) of the elderly were considered insufficiently active. Moreover, among individuals that did not reported DM, the prevalence of $P A$ insufficient was 82.5\% (95\% CI 81.9-83.2) and 86.3\% (95\% CI 85.4-87.2) respectively. It is high the prevalence of physical inactivity among individuals who report or not the DM.
\end{abstract}

\section{Keywords}

Diabets, Motor activity, epidemiology.
Rev Bras Ativ Fis Saúde p. 519-531 DOI: http://dx.doi.org/10.12820/23171634.2012v17n6p519

1 Programa de Pós-graduação em Educação Física, Escola Superior de Educação Física, Universidade Federal de Pelotas, Pelotas, RS, Brasil.

2 Faculdade de Medicina, Departamento de Medicina Social, Universidade Federal de Pelotas, Pelotas, RS, Brasil.

3 Faculdade de Enfermagem, Universidade Federal de Pelotas, Pelotas, RS, Brasil.

4 Programa de Pós-graduação em Epidemiologia, Universidade Federal de Pelotas, Pelotas, RS, Brasil.

5 Faculdade de Terapia Ocupacional, Universidade Federal de Pelotas, Pelotas, RS, Brasil. 


\section{INTRODUÇÃO}

Segundo a Organização Mundial da Saúde, existem cerca de 347 milhões de portadores de Diabetes Mellitus (DM) no mundo, o que permite dizer que o número estimado é muito superior às projeções que existiam para o ano 2010, segundo as quais, seriam de 285 milhões de casos. ${ }^{1} \mathrm{O}$ DM faz parte de um grupo de doenças e agravos não transmissíveis (DANTs), que representam a principal causa de morte, tanto no cenário internacional, quanto nacional. No Brasil, $72 \%$ das mortes em 2007 foram por DANTs. ${ }^{2}$ Em 2009, o Sistema de Informações sobre Mortalidade do Ministério da Saúde, notificou 52.104 mortes por DM no país. ${ }^{3}$

Entre os muitos fatores de risco para o DM citados na literatura, alguns têm por característica o fato de não poderem ser modificados, entre eles a raça, a idade e a história familiar. No entanto, existem outros fatores considerados modificáveis: o nível de AF, o índice de massa corporal (IMC), a deposição central de gordura e o padrão dietético. Este grupo de fatores, considerados modificáveis, são pontos passíveis de intervenção para reverter o processo epidêmico de surgimento do DM. ${ }^{4}$ Corroborando com afirmação de que os fatores modificáveis são importantes na reversão do aparecimento da doença, o estudo de Knowler et al. (2002)5, uma intervenção com indivíduos em risco de desenvolver o DM, baseado em prática de $\mathrm{AF}$ e aconselhamento nutricional, apontou resultados positivos na diminuição do aparecimento da doença. No mesmo sentido, o Diabetes Prevention Program (DPP) demonstrou que intervenção no estilo de vida (dieta e exercício) produziu perda de $7 \%$ de peso, aumento na AF para 150 minutos por semana, e reduziu a incidência de DM em 58\% no grupo intervenção e em 31\% no grupo tratado com metformina em comparação ao grupo controle. ${ }^{6}$

No Brasil, estudos recentes mostraram que 5,8\% da população com 18 anos ou mais, tiveram diagnóstico de DM, enquanto alguns dados referentes aos fatores modificáveis indicaram, que entre os brasileiros, mais de $85 \%$ são insuficientemente ativos no lazer. ${ }^{4,7}$ Em relação ao excesso de peso, o percentual passou de 47,2\% para 52,6\% nos últimos seis anos nos homens. ${ }^{8}$

Portanto, entendendo que a utilização do autorrelato sobre o diagnóstico de DM é adequado para estudos epidemiológicos ${ }^{9,10,11}$ e que a prática da $\mathrm{AF}$ é um, entre outros fatores modificáveis importantes, o presente artigo tem o objetivo de conhecer a prevalência de autorrelato de $\mathrm{DM}$ e verificar a prática de $\mathrm{AF}$ na população, assim como de algumas variáveis associadas com os adultos e idosos brasileiros.

\section{MÉTODOS}

Foi realizado um inquérito epidemiológico, com delineamento transversal e de base populacional, em uma amostra independente de adultos (20 a 59 anos) e idosos (60 anos ou mais) moradores da zona urbana de 100 municípios das cinco regiões geográficas do Brasil. O estudo compreendeu 23 estados Brasileiros: Acre, Alagoas, Bahia, Ceará, Espírito Santo, Goiás, Maranhão, Mato Grosso, Mato Grosso do Sul, Minas Gerais, Pará, Paraíba, Paraná, Pernambuco, Piauí, Rio de Janeiro, Rio Grande do Norte, Rio Grande do Sul, Rondônia, Santa Catarina, São Paulo, Sergipe e Tocantins. Todos os municípios brasileiros foram listados em ordem crescente de acordo com o porte populacional e sorteados. $\mathrm{O}$ resultado do sorteio determinou a composição dos estados participantes da amostra.

A amostra foi localizada em múltiplos estágios, considerando: 1) o porte populacional dos municípios brasileiros em cinco estratos: a) menos de $10 \mathrm{mil} \mathrm{ha-}$ 
bitantes, b) de 10 mil a menos de 20 mil habitantes, c) de 20 mil a menos de 100 mil habitantes, d) de 100 mil a menos de 1 milhão de habitantes e, e) de 1 milhão de habitantes ou mais, 2) os municípios, 3) os setores censitários, 4) os domicílios e, 5) os elegíveis residentes nos domicílios, conformando um marco de amostras complexas em múltiplos níveis de unidades amostrais. ${ }^{12,13,14}$ Os indivíduos constituíram a unidade de análise do estudo, e as amostras de adultos e idosos foram localizadas de forma independente em cada setor censitário.

Para a seleção dos municípios e setores censitários urbanos, foi adotado a divisão territorial e a malha setorial utilizada no Censo Demográfico 2000, do Instituto Brasileiro de Geografia e Estatística (IBGE). ${ }^{15}$ Todos os indivíduos adultos e idosos, moradores em domicílios particulares, localizados nos setores censitários urbanos selecionados, responderam ao instrumento que avaliou questões socioeconômicas, demográficas e de saúde, relacionadas ao estudo.

A coleta de dados foi realizada por 55 entrevistadores especialmente treinados por mais de 40 horas, que utilizaram um "Personal Digital Assistant" (PDA). Após coletados, os dados eram armazenados e repassados para a coordenação do estudo na Universidade Federal de Pelotas via internet. Cinco por cento de todas as entrevistas foram sorteadas para o controle de qualidade, realizado pelo supervisor de campo, em no máximo três dias após a primeira entrevista de forma face-aface. Outra estratégia utilizada para o controle de qualidade foi a comparação dos dados obtidos pelo PDA em relação às coordenadas geográficas de cada domicílio, com a localização no mapa do setor censitário definido pelo IBGE, com o objetivo de acompanhar a distribuição da amostra em relação à área do setor, diminuindo assim a chance de viés de seleção.

O questionário utilizado foi padronizado e pré-testado e a operacionalização do desfecho deu-se através da pergunta: "Algum médico lhe disse que o $\operatorname{Sr}(\mathrm{a})$ tem diabetes (açúcar alto no sangue)?”. Embora o estudo não tenha investigado o diagnóstico médico de DM, a utilização do autorrelato tem se mostrado importante em estudos epidemiológicos, devido as dificuldades técnicas e aos altos custos nas grandes investigações populacionais. ${ }^{9,10,11}$ Neste sentido, a utilização da estratégia de autorrelato, tem demonstrado alta sensibilidade e especificidade, comprovando a validade de utilização do relato para essa doença e outros acontecimentos em saúde. ${ }^{16,17}$

Para a definição de AF foi utilizado à seção 2 da versão longa do Questionário Internacional de Atividade Física (IPAQ) com as questões relacionadas ao domínio do lazer. ${ }^{18,19}$

As variáveis independentes incluídas na análise foram: sexo; idade em anos (20 a 29, 30 a 39, 40 a 49, 50 a 59, 60 a 69, 70 a 79, 80 ou mais); cor da pele (branca e não branca), nível socioeconômico (NSE) (A,B,C, D e E), porte dos municípios ( $<10$ mil, 10 a $<20$ mil, 20 a $<100$ mil, 100 mil a $<1$ milhão e 1milhão ou mais), atividade física ( $\mathrm{sim}: \geq 150 \mathrm{~min} / \mathrm{sem}$ e não: $\leq 149 \mathrm{~min} / \mathrm{sem}$ ), consulta médica no último ano por DM (sim e não), índice de massa corporal (IMC) (14 a 25: normal; 25,1 a 30: sobrepeso; 30,1 ou mais: obesidade) e diagnóstico médico referido de hipertensão arterial sistêmica (HAS) (sim e não). Também foram utilizadas variáveis referentes ao recebimento de orientações no último ano para: comer pouco sal (sim e não), pouco açúcar (sim e não), pouca gordura (sim e não), para manter o peso ideal (sim e não) e para fazer AF (sim e não).

As análises descritivas incluíram cálculos de médias com respectivos desvios padrão, e de proporções com intervalos de confiança de $95 \%$. Na análise univariada, a prevalência de DM foi calculada para cada grupo das variáveis independentes e o nível de significância foi verificado usando os testes de Wald para heterogeneidade e 
tendência linear. A análise com ajuste robusto para variância foi realizada por regressão de Poisson, com cálculo das razões de prevalência ajustadas, intervalo de confiança de $95 \%$ e valores de significância conforme os testes descritos anteriormente. ${ }^{20}$

As análises levaram em consideração o modelo de análise que incluiu as variáveis independentes, sexo, idade e cor da pele no primeiro nível, IMC e diagnóstico médico referido de HAS no segundo nível. A prática de AF no lazer e o recebimento de orientações relacionadas a saúde foram estudas em adultos e idosos com e sem autorrelato de DM, portanto caracterizando neste ponto a utilização da variável desfecho como uma variável exploratória.

Este estudo é parte de um projeto que investigou vários outros desfechos em saúde, coordenado pelo Departamento de Medicina Social da Universidade Federal de Pelotas pelo Grupo AQUARES. O Comitê de Ética da Faculdade de Medicina da Universidade Federal de Pelotas aprovou o protocolo do estudo, sob o número 152/07 de 23 de novembro de 2007, e consentimento informado foi obtido de todos os participantes. Os autores declararam não haver conflito de interesse no presente estudo.

\section{RESULTADOS}

Foram visitados 638 setores censitários de 23 estados brasileiros. Do total de adultos (12.402) e de idosos (6.616) entrevistados, 12.344 adultos e 6.582 idosos responderam a questão referente à $\mathrm{DM}$, caracterizando uma perda de $0,5 \%$ em cada um dos grupos. Com a amostra final, a margem de erro para a estimativa da prevalência de DM entre adultos foi de 1.2 ponto percentual (pp) e de $1.3 \mathrm{pp}$ entre os idosos. Para avaliação das associações o estudo teve o poder de $80 \%$ para identificar razões de prevalências de 1,2 ou superiores (adultos) e 1,3 ou superiores (idosos) com nível de confiança de 95\%.

A prevalência de autorrelato de $\mathrm{DM}$, por ter recebido algum dia o diagnóstico de um médico, na população de adultos foi de 3,5\% (IC95\% 3,2-3,9) enquanto nos idosos foi de 16,9\% (IC95\% 15,9-17,8).

Em relação às regiões do país, a prevalência em adultos foi de 2,8\% (IC95\% 1,7-3,8) na região Norte, 2,9\% (IC95\% 2,3-3,5) na região Nordeste, 3,6\% (IC95\% 3,1-4,2) na região Sudeste, 4,0\% (IC95\% 3,3-4,8) na região Sul e de 4,4\% (IC95\% $3,3-5,5)$ na região Centro-Oeste. Os valores caracterizaram a diferença das regiões Norte e Nordeste em relação a todas demais. $(\mathrm{P}=0,03)$.

Entre os idosos os valores foram 14,2\% (IC95\% 11,3-17,1) na região Norte, $15,7 \%$ (IC95\% 13,8-17,5) na região Nordeste, 17,6\% (IC95\% 16,1-19,2) na região Sudeste, 18,4\% (IC95\% 16,5-20,4) na região Sul e de 15,5\% (IC95\% 12,9$18,2)$ na região Centro Oeste $(\mathrm{P}=0,07)$.

Considerando o porte populacional, a prevalência de autorrelato de DM variou de $3,4 \%$ a $4,5 \%$ nos adultos $(\mathrm{P}=0,66)$ e de $12 \%$ a $19 \%$ nos idosos $(\mathrm{P}=0,001)$. A Figura $1 \mathrm{~A}$ e B, apresenta as prevalências conforme os portes populacionais estudados.

A Tabela 1, descreve a amostra de adultos e idosos conforme as variáveis utilizadas nas análises do estudo. Entre os adultos, o sexo feminino foi o mais prevalente, assim como os indivíduos na faixa dos 20 à 29 anos de idade. Grande parte da população é da cor de pele não branca e mais da metade pertence ao NSE C. Atividade física insuficiente no lazer foi de $82,5 \%$. Em relação ao recebimento de orientações no último ano voltadas à uma vida saudável, as prevalências encontradas variaram de $26,4 \%$ para manter o peso ideal a $38,2 \%$ para comer pouca gordura. 
Tabela 1 - Descrição da amostra de adultos e idosos de acordo com as variáveis utilizadas no estudo. UFPel, AQUARES, 2009.

\begin{tabular}{|c|c|c|c|c|c|c|}
\hline \multirow[b]{2}{*}{ Variáveis } & \multicolumn{3}{|c|}{ Adultos } & \multicolumn{3}{|c|}{ Idosos } \\
\hline & $\mathrm{N}$ total & $\mathrm{N}$ & $\%$ & $\mathrm{~N}$ total & $\mathrm{N}$ & $\%$ \\
\hline Sexo & 12.402 & & & 6.616 & & \\
\hline Masculino & & 5.574 & 44.9 & & 2.714 & 41.0 \\
\hline Feminino & & 6.828 & 55.1 & & 3.902 & 59.0 \\
\hline Idade & 12.371 & & & 6.601 & & \\
\hline $20-29$ & & 3.938 & 31.8 & & & \\
\hline $30-39$ & & 3.114 & 25.2 & & & \\
\hline $40-49$ & & 2.958 & 23.9 & & & \\
\hline $50-59$ & & 2.361 & 19.1 & & & \\
\hline $60-69$ & & & & & 3.308 & 50.1 \\
\hline $70-79$ & & & & & 2.251 & 34.1 \\
\hline 80 ou mais & & & & & 1.042 & 15.8 \\
\hline Cor da pele & 11.979 & & & 6.456 & & \\
\hline Branca & & 4.752 & 39.7 & & 2.604 & 40.3 \\
\hline Não branca & & 7.227 & 60.3 & & 3.852 & 59.7 \\
\hline Nível socioeconômico & 11.674 & & & 6.346 & & \\
\hline$A / B$ & & 3.302 & 28.3 & & 1.079 & 17.0 \\
\hline C & & 6.013 & 51.5 & & 2.650 & 41.8 \\
\hline $\mathrm{D} / \mathrm{E}$ & & 2.359 & 20.2 & & 2.617 & 41.2 \\
\hline Sedentário no Lazer & 12.303 & & & 6.594 & & \\
\hline Não & & 2.147 & 17.5 & & 887 & 13.5 \\
\hline Sim & & 10.156 & 82.5 & & 5.707 & 86.5 \\
\hline IMC & 8.086 & & & 5.303 & & \\
\hline Normal & & 4.064 & 50.3 & & 2.310 & 43.6 \\
\hline Sobrepeso & & 2.535 & 31.3 & & 1.948 & 36.7 \\
\hline Obeso & & 1.487 & 18.7 & & 1.045 & 19.7 \\
\hline Diag. médico referido HAS & 12.324 & & & 6.580 & & \\
\hline Não & & 10.320 & 83.7 & & 3.168 & 48.2 \\
\hline Sim & & 2.004 & 16.3 & & 3.412 & 51.8 \\
\hline Orient. manter o peso ideal & 12.265 & & & 6.577 & & \\
\hline Não & & 9.033 & 73.5 & & 4.774 & 72.6 \\
\hline Sim & & 3.232 & 26.4 & & 1.803 & 27.4 \\
\hline Orient. comer pouco sal & 12.283 & & & 6.595 & & \\
\hline Não & & 7.831 & 63.7 & & 2.599 & 39.4 \\
\hline Sim & & 4.452 & 36.3 & & 3.996 & 60.6 \\
\hline Orient. comer pouco açucar & 12.287 & & & 6.586 & & \\
\hline Não & & 8.766 & 71.3 & & 3.699 & 56.2 \\
\hline Sim & & 3.521 & 28.7 & & 2.887 & 43.8 \\
\hline Orient. comer pouca gordura & 12.282 & & & 6.595 & & \\
\hline Não & & 7.596 & 61.8 & & 2.752 & 41.7 \\
\hline Sim & & 4.686 & 38.2 & & 3.843 & 58.3 \\
\hline Orient. fazer AF & 12.282 & & & 6.577 & & \\
\hline Não & & 8.572 & 69.8 & & 4.588 & 69.8 \\
\hline Sim & & 3.710 & 30.2 & & 1.989 & 30.2 \\
\hline
\end{tabular}

No grupo dos idosos, as mulheres também são maioria e prevalece a faixa dos 60 a 69 anos. Assim como nos adultos, a cor de pele predominante foi a não branca e o NSE mais prevalente o C. Atividade física insuficiente foi de $86,5 \%$ na amostra de idosos e as orientações variaram de $27,4 \%$ para manter o peso ideal a $60,6 \%$ para comer pouco sal. 

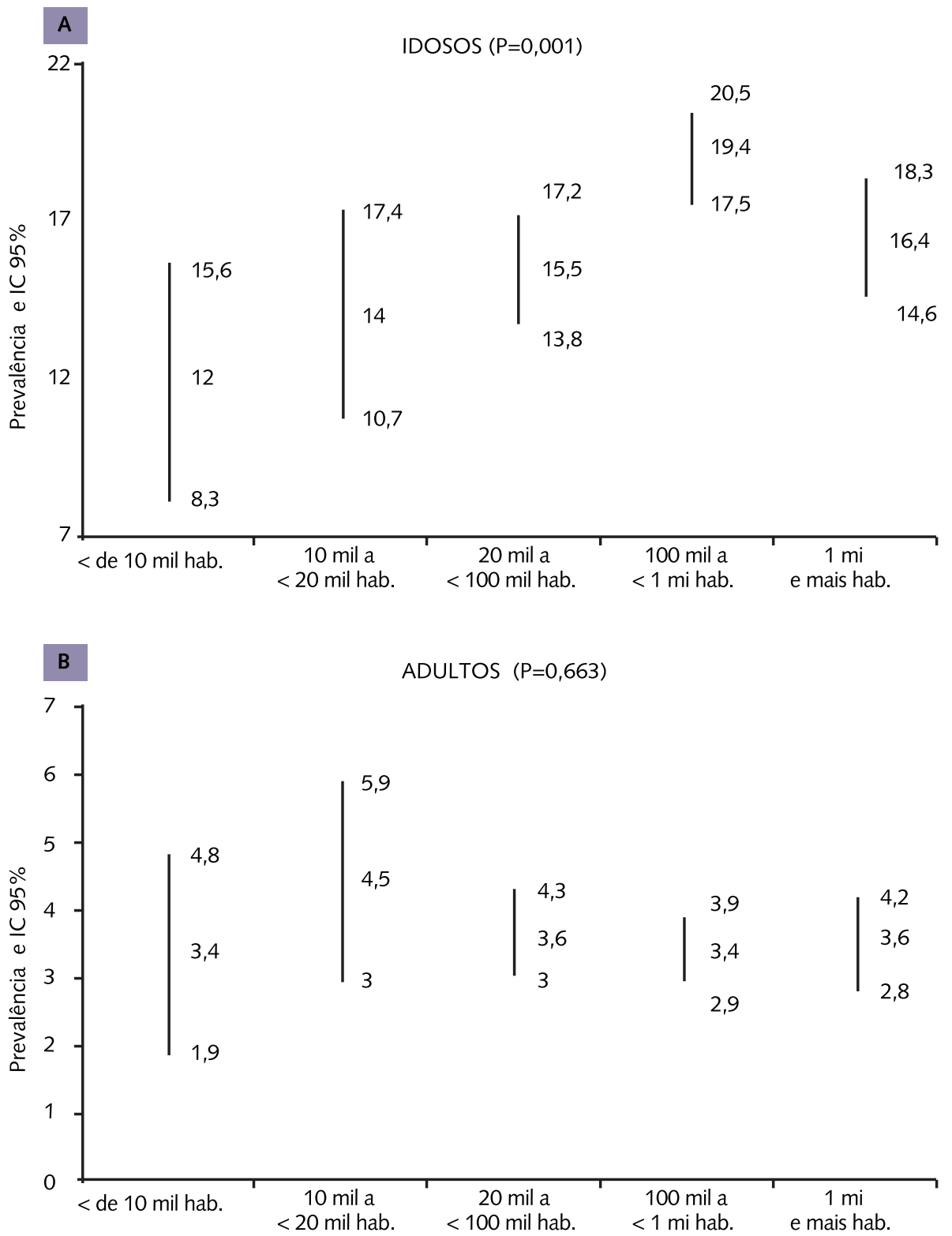

Figura 1 - Prevalência e IC $95 \%$ de autorrelato de Diabetes em idosos e adultos de acordo com o porte dos municípios brasileiros. UFPel, AQUARES, 2009. (Linha Preta Idosos $P=0,001$; Linha Cinza Adultos $\mathrm{P}=0,663)$.

A Tabela 2 apresenta as análises, bruta e ajustada para a associação de autorrelato de DM em adultos e as variáveis independentes utilizadas no estudo. Foi encontrada associação na análise bruta e ajustada, com maior risco no sexo feminino, nos considerado com obesidade e naqueles indivíduos que receberam no último ano diagnóstico de HAS. A variável sexo foi a única das associadas nas análises bruta e ajustada que ganhou em magnitude quando controlada para as demais variáveis. Todas as demais variáveis diminuíram a magnitude da associação, quando ajustadas no modelo de análise. A variável NSE, mudou seu status de não associada para associada, com risco aumentado para o NSE D e E, após controlada para as demais. Por fim, no grupo de adultos, a idade mostrou associação na análise bruta e ajustada com tendência a maiores riscos conforme aumenta a idade.

A Tabela 3 apresenta a mesma análise anterior, porém para o grupo de idosos. Associação nas análises bruta e ajustada foi encontrada para as variáveis sexo, 
obesidade e diagnóstico médico referido de HAS. A variável sexo mostrou risco aumentado quando ajustadas as demais variáveis dos diferentes níveis de análise.

Em relação à prática de $\mathrm{AF}$, observou-se que entre os indivíduos que relataram ter DM, 82,6\% (IC95\% 79,0-86,1) dos adultos e 88,2\% (IC95\% 86,3-90,1) dos idosos foram considerados insuficientemente ativos. Analisando a AF insuficiente entre os indivíduos que relatam não ter $\mathrm{DM}$, os valores encontrados para a prevalência foram de 82,5\% (IC95\% 81,9-83,2) e 86,3\% (IC95\% 85,4-87,2) respectivamente para adultos e idosos. Os resultados mostram que não existe diferença significativa em relação à $\mathrm{AF}$, para os indivíduos adultos ou idosos que relataram ter $\mathrm{DM}$ e não ter $\mathrm{DM}(\mathrm{P}=0,99$ adultos e $\mathrm{P}=0,10$ idosos).

Quando estudado o nível de AF dos indivíduos que relataram DM e, que foram considerados insuficientemente ativos $(82,6 \%)$, entre os adultos, verificou-se que $72,5 \%$ não realizam nenhuma $\mathrm{AF}$ no lazer e 10,1\% realiza $\mathrm{AF}$ entre 10 e 149 minutos por semana. No intervalo de tempo de 100 a 149 minutos de AF/semana no lazer, encontramos que $3,9 \%$ dos adultos estão neste grupo.

Os mesmos resultados para o grupo de idosos mostrou que $77,4 \%$ não realizam AF e 10,8\% estão no intervalo de tempo entre 10 e 149 minutos de AF/ semana. A prevalência de idosos que realiza alguma AF entre 100 e 149 minutos/ semana foi de $4,3 \%$.

A Tabela 4 apresenta os resultados relacionados ao recebimento de orientações no sentido de comer pouco sal, manter o peso ideal, comer pouco açúcar, comer pouca gordura e fazer AF. Os resultados mostram que para adultos e idosos, todas as variáveis mostraram diferença significativa a favor do recebimento destas orientações, para aqueles que autorrelataram diagnóstico médico de DM.

Tabela 2 - Prevalência, análises bruta e ajustada para associação entre autorrelato de Diabetes e as variáveis socioeconômicas, demográficas e de saúde entre adultos de 23 estados do Brasil. UFPel, AQUARES, 2009

\begin{tabular}{|c|c|c|c|c|c|}
\hline \multirow[b]{2}{*}{ Variáveis } & \multicolumn{2}{|c|}{ Análise bruta } & \multirow[b]{2}{*}{$p$} & \multicolumn{2}{|c|}{ Análise ajustada } \\
\hline & $\%$ & $\mathrm{RP}(\mathrm{IC95 \% )}$ & & $\mathrm{RP}(\mathrm{IC} 95 \%)$ & $\mathrm{p}$ \\
\hline Sexo & & & 0.005 & & 0.004 \\
\hline Masculino & 3.0 & 1 & & 1 & \\
\hline Feminino & 4.0 & $1.31(1.08-1.58)$ & & $1.33(1.09-1.61)$ & \\
\hline Idade & & & $0.000^{\top}$ & & $0.000^{\top}$ \\
\hline $20-29$ & 1.3 & 1 & & 1 & \\
\hline $30-39$ & 1.6 & $1.22(0.82-1.79)$ & & $1.53(1.00-2.33)$ & \\
\hline $40-49$ & 4.4 & $3.32(2.41-4.57)$ & & $4.26(2.98-6.09)$ & \\
\hline $50-59$ & 8.7 & $6.59(4.89-8.91)$ & & $8.44(6.01-11.8)$ & \\
\hline Cor da pele & & & 0.69 & & 0.52 \\
\hline Branca & 3.5 & 1 & & 1 & \\
\hline Não branca & 3.6 & $1.03(0.86-1.26)$ & & $1.07(0.87-1.32)$ & \\
\hline IMC & & & $0.000^{\top}$ & & $0.000^{\top}$ \\
\hline Normal & 2.2 & 1 & & 1 & \\
\hline Sobrepeso & 5.0 & $2.30(1.76-3.00)$ & & $1.43(1.08-1.90)$ & \\
\hline Obeso & 7.3 & $3.38(2.57-4.45)$ & & $1.71(1.28-2.29)$ & \\
\hline Diag. médicc & & & $0.000^{\top}$ & & 0.000 \\
\hline Não & 1.9 & 1 & & 1 & \\
\hline Sim & 11.9 & $6.18(5.16-7.42)$ & & $3.21(2.49-4.13)$ & \\
\hline
\end{tabular}

T - Teste de Wald para Tendência Linear. 
Tabela 3 - Prevalência, análises bruta e ajustada para associação entre autorrelato de Diabetes e as variáveis socioeconômicas, demográficas e de saúde entre idosos de 23 estados do Brasil. UFPel, AQUARES, 2009.

\begin{tabular}{|c|c|c|c|c|c|}
\hline & & Análise bruta & & Análise ajustada & \\
\hline Variáveis & $\%$ & $\mathrm{RP}(\mathrm{IC95} \%)$ & $\mathrm{p}$ & $\mathrm{RP}(\mathrm{IC95 \%})$ & $\mathrm{p}$ \\
\hline Sexo & & & 0.000 & & 0.000 \\
\hline Masculino & 14.0 & 1 & & 1 & \\
\hline Feminino & 18.9 & $1.35(1.20-1.51)$ & & $1,36(1.21-1.52)$ & \\
\hline Idade & & & $0.68^{\top}$ & & $0.65^{\top}$ \\
\hline $60-69$ & 16.6 & 1 & & 1 & \\
\hline $70-79$ & 18.0 & 1.08 & & $1.07(0.95-1.21)$ & \\
\hline 80 ou mais & 15.3 & 0.92 & & $0.91(0.77-1.08)$ & \\
\hline Cor da pele & & & 0.416 & & 0.32 \\
\hline Branca & 16.5 & 1 & & 1 & \\
\hline Não branca & 17.3 & $1.05(0.94-1.17)$ & & $1.06(0.9-1.19)$ & \\
\hline IMC & & & 0.000 & & 0.000 \\
\hline Normal & 11.3 & 1 & & 1 & \\
\hline Sobrepeso & 19.1 & $1.68(1.45-1.95)$ & & $1.55(1.33-1.81)$ & \\
\hline Obeso & 24.7 & $2.18(1.87-2.55)$ & & $1.81(1.53-2.14)$ & \\
\hline \multicolumn{2}{|c|}{ Diag. médico referido HAS } & & 0.000 & & 0.000 \\
\hline Não & 9.7 & 1 & & 1 & \\
\hline Sim & 23.4 & $2.41(2.13-2.72)$ & & $2.13(1.84-2.46)$ & \\
\hline
\end{tabular}

T - Teste de Wald para Tendência Linear.

Tabela 4 - Prevalência de variáveis relacionadas ao aconselhamento entre adultos e idosos, com e sem autorrelato de diabetes no Brasil. UFPel, AQUARES, 2009.

\begin{tabular}{|c|c|c|c|c|c|c|c|}
\hline \multirow[b]{2}{*}{ Variáveis } & \multicolumn{3}{|c|}{ Não diabéticos } & \multicolumn{4}{|c|}{ Diabéticos } \\
\hline & $\mathrm{N}$ total & $\mathrm{N}$ & $\%$ & $\mathrm{~N}$ total & $\mathrm{N}$ & $\%$ & $p$ \\
\hline \multicolumn{8}{|l|}{ ADULTOS } \\
\hline $\begin{array}{l}\text { Orient. para fazer } \\
\text { atividade física }\end{array}$ & 12.282 & & & 434 & & & $<0.001$ \\
\hline Não & & 8.572 & 69.79 & & 184 & 42.40 & \\
\hline Sim & & 3.710 & 30.21 & & 250 & 57.60 & \\
\hline $\begin{array}{l}\text { Orient. para comer } \\
\text { pouco sal }\end{array}$ & 12.283 & & & 436 & & & $<0.001$ \\
\hline Não & & 7.831 & 63.75 & & 122 & 27.89 & \\
\hline Sim & & 4.452 & 36.25 & & 314 & 72.02 & \\
\hline $\begin{array}{l}\text { Orient. para comer } \\
\text { pouco açúcar }\end{array}$ & 12.287 & & & 437 & & & $<0.001$ \\
\hline Não & & 8.766 & 71.34 & & 96 & 21.92 & \\
\hline Sim & & 3.521 & 28.66 & & 338 & 78.08 & \\
\hline $\begin{array}{l}\text { Orient. para comer } \\
\text { pouca gordura }\end{array}$ & 12.282 & & & 436 & & & $<0.001$ \\
\hline Não & & 7.596 & 61.85 & & 98 & 22.48 & \\
\hline Sim & & 4.686 & 38.61 & & 338 & 77.52 & \\
\hline $\begin{array}{l}\text { Orient. para manter o } \\
\text { peso ideal }\end{array}$ & 12.265 & & & 438 & & & $<0.001$ \\
\hline Não & & 9.033 & 73.65 & & 190 & 43.38 & \\
\hline Sim & & 3.232 & 26.35 & & 248 & 56.62 & \\
\hline
\end{tabular}




\begin{tabular}{|c|c|c|c|c|c|c|c|}
\hline \multirow[b]{2}{*}{ Variáveis } & \multicolumn{3}{|c|}{ Não diabéticos } & \multicolumn{4}{|c|}{ Diabéticos } \\
\hline & $\mathrm{N}$ total & $\mathrm{N}$ & $\%$ & $\mathrm{~N}$ total & $\mathrm{N}$ & $\%$ & $\mathrm{p}$ \\
\hline \multicolumn{8}{|l|}{ IDOSOS } \\
\hline $\begin{array}{l}\text { Orient. fazer atividade } \\
\text { física }\end{array}$ & 6.577 & & & 1.101 & & & $<0.001$ \\
\hline Não & & 4.588 & 69.76 & & 622 & 56.49 & \\
\hline Sim & & 1.989 & 30.24 & & 479 & 43.51 & \\
\hline Orient. comer pouco sal & 6.595 & & & 1.106 & & & $<0.001$ \\
\hline Não & & 2.599 & 39.41 & & 236 & 21.34 & \\
\hline Sim & & 3.996 & 60.59 & & 870 & 78.66 & \\
\hline $\begin{array}{l}\text { Orient. comer pouco } \\
\text { açúcar }\end{array}$ & 6.586 & & & 1.108 & & & $<0.001$ \\
\hline Não & & 3.699 & 56.16 & & 181 & 16.34 & \\
\hline Sim & & 2.887 & 43.84 & & 927 & 83.86 & \\
\hline $\begin{array}{l}\text { Orient. comer pouca } \\
\text { gordura }\end{array}$ & 6.595 & & & 1.107 & & & $<0.001$ \\
\hline Não & & 2.752 & 41.73 & & 232 & 20.96 & \\
\hline Sim & & 3.843 & 58.27 & & 875 & 79.04 & \\
\hline $\begin{array}{l}\text { Orient. manter o peso } \\
\text { ideal }\end{array}$ & 6.577 & & & 1.101 & & & $<0.001$ \\
\hline Não & & 4.774 & 72.59 & & 640 & 58.13 & \\
\hline Sim & & 1.803 & 27.41 & & 461 & 41.87 & \\
\hline
\end{tabular}

\section{DISCUSSÃO}

A prevalência de autorrelato de DM encontrada foi 3,5\% para adultos e 16,9\% para idosos. Os resultados deste estudo foram menores em relação à pesquisa VIGITEL realizada no Brasil em 2011 com mais de 54 mil entrevistas telefônicas, que encontrou prevalências maiores, de 5,6\% nos adultos de 18 a 64 anos e $21,6 \%$ dos idosos com 65 anos ou mais. ${ }^{8}$ Acreditamos que três fatores podem contribuir para as diferenças: a) os grupos etários estudados, pois neste estudo foram incluímos adultos de 20 a 59 anos enquanto o estudo do VIGITEL consideraram grupo etário de 18 a 64 anos, portanto cerca de 13\% mais amplo, o que pode ter determinado a maior prevalência. $\mathrm{O}$ mesmo ocorreu no caso dos idosos, que foram considerados a partir de 60 anos em nosso estudo e 65 no VIGITEL; b) o fato de que a pesquisa VIGITEL foi realizada apenas em capitais e, de acordo com nossos resultados, há uma tendência de aumento no número de diabéticos de acordo com o porte do município. Em municípios considerados grandes, caso da maioria das capitais, foi encontrada a maior prevalência (19\%) de autorrelato de DM em idosos, com diferença significativa entre os portes $(\mathrm{p}=0,001)$. Sendo assim, o fato de nosso estudo ter uma amostra representativa dos vários portes de municípios do Brasil, pode estar determinando esta diferença encontrada nas prevalências de DM entre os adultos e idosos e; c) Além disso, o VIGITEL é um inquérito realizado pelo telefone, sendo este um bem dos mais prevalente nos maiores níveis socioeconômicos, e provavelmente, indivíduos pertencentes a estas categorias tem mais acesso à consultas médicas e consequentemente ao diagnóstico de DM. Outra hipótese deste estudo é de que o aumento da população com DM nos municípios de maiores portes, pode estar relacionado ao maior acesso às consultas e exames, que muitas vezes não estão disponíveis nos municípios menores, os quais são maioria no Brasil.

Maiores prevalências de autorrelato de DM entre adultos foram encontradas nas regiões Centro-oeste $(4,41 \%)$ e $\mathrm{Sul}(4,04 \%)$, e as menores nas regiões Norte 
$(2,8 \%)$ e Nordeste $(2,9 \%)$. Estes resultados são semelhantes aos encontrados em um estudo transversal de base populacional, realizado no Brasil em 2008 (Pesquisa Nacional por Amostra de Domicílios), que mostrou existir mais indivíduos com diagnóstico médico referido de DM nas regiões Sudeste (5,0\%) e Sul (4,5\%) e menores prevalências nas regiões Norte $(3,3 \%)$ e Nordeste $(3,7 \%) .{ }^{21}$ Nossa hipótese para este achado está relacionada as diferenças entre os padrões alimentares das regiões mencionada.

No presente artigo, o autorrelato de DM entre os adultos, esteve associado com risco aumentado no sexo feminino, com obesidade e diagnóstico médico referido de HAS, além de apresentar tendência de aumento da prevalência com o aumento da idade, concordando com os resultados da PNAD 2008, VIGITEL 2011, com o estudo de Francisco e colaboradores. ${ }^{8,215,22}$

O sexo feminino apresentou maior risco para autorrelato de $\mathrm{DM}$ e dados do Ministério da Saúde de maio de 2012, fortalecem nossos achados revelando que no Brasil, a população adulta de mulheres atingida pela doença (6\%) é maior que homens $(5,2 \%) .{ }^{8}$ Acreditamos que a maior prevalência de autorrelato do diagnóstico de DM no sexo feminino pode ser resultado da melhor percepção da mulher quanto aos sintomas e sinais físicos das doenças, do fato de consultar mais que os homens, do conhecimento adquirido desempenhando o papel de cuidadora da família, bem como da realização de mais exames diagnósticos por esse grupo, além das próprias características biológicas. ${ }^{23,24,25}$

O aumento de peso é um dos grandes vilões na propagação da doença, pois o DM apresenta forte associação com excesso de peso. ${ }^{26} \mathrm{~A}$ população brasileira acima do peso aumentou de 43\% para 49\% em cinco anos e, em 2011, mais da metade da população brasileira estava acima do peso. ${ }^{8}$ Uma vez que a obesidade aumenta no mundo todo, o DM tende a piorar. ${ }^{26}$

A associação do autorrelato de DM com o diagnóstico de HAS concorda com a literatura, que diz que esta patologia é mais frequente em indivíduos portadores de DM, quando comparados com a população em geral. ${ }^{23}$ Entre os anos de 2005 e 2008, 67\% dos adultos americanos com diagnóstico médico referido de DM apresentaram níveis elevados de pressão arterial. ${ }^{27,28}$

Em relação à $\mathrm{AF}$ no lazer, a prevalência de indivíduos insuficientemente ativos foi de $82,6 \%$ e $88,2 \%$ entre os adultos e idosos com autorrelato de DM, respectivamente. Estes resultados discordam do estudo de Duarte e colaboradores em 2012 29 , que encontrou em idosos com DM, moradores de Porto Alegre, uma prevalência de insuficientemente ativos de cerca 40\%, quando avaliados pelo IPAQ. ${ }^{29}$ Provavelmente esta diferença se deva ao fato de que o estudo de Duarte e colaboradores $(2012)^{29}$ foi realizado utilizando todos os domínios do IPAQ em sua versão longa, enquanto neste estudo foi considerada somente a sessão de $\mathrm{AF}$ durante o lazer.

Em outro estudo realizado com idosos portadores de DM, e participantes de uma associação municipal no estado de São Paulo, $12,9 \%$ da amostra foi considerada insuficientemente ativa quando avaliada pelo IPAQversão curta. ${ }^{30}$ Nota-se que no estudo mencionado a amostra foi de idosos que participavam de grupo de $\mathrm{DM}$ e, portanto, certamente existia neste contexto, o estímulo para aumentar a AF como forma de prevenir e tratar a doença.

Os resultados apresentados mostram que não foi observada diferença significativa na variável $\mathrm{AF}$, quando comparados aqueles que autorrelataram DM e os que não a relataram ( $\mathrm{p}=0,99 \mathrm{em}$ adultos e $\mathrm{p}=0,10 \mathrm{em}$ idosos). Este resultado está de acordo com o estudo de Florindo e colaboradores em $2009^{7}$, que analisou as entrevistas do VIGITEL realizadas em 2006, e encontrou 85,2\% de indivíduos insu- 
ficientemente ativos no lazer. ${ }^{7}$ Outros estudos nacionais e internacionais mostram que a prevalência de inatividade física é alta na população em geral. ${ }^{31,32,33}$ Os dados mais recentes mostram que no mundo 31,1\% (IC95\% 30,9 -31,2) dos adultos são fisicamente inativos, com proporções que variam de 17\% (IC95\% 16,8 -17,2) no sudeste da Ásia até cerca de 43\% nas Américas e no Mediterrâneo oriental. ${ }^{34}$ Desta forma, pelos resultados apresentados para a população que autorrelata DM e para a população geral, podemos inferir que o fato do indivíduo ter ou não DM, não determina maior comprometimento para a realização de $\mathrm{AF}$, um dos fatores importantes e modificáveis, relacionado a doença, que certamente beneficia o portador.

Em relação ao recebimento de orientações, verifica-se que os indivíduos que autorrelataram diagnóstico médico de DM, mostram que receberam mais orientações em relação aos que não autorrelataram a doença. Atribuímos isso ao fato de que ao receber o diagnóstico de $\mathrm{DM}$, o indivíduo também recebe orientações, pois necessita cuidar da alimentação, assim como, praticar AF, para controle e tratamento da doença. ${ }^{35}$

Os dados apresentados neste artigo contribuem para o conhecimento da realidade dos portadores de DM no Brasil, pois apresenta uma amostra com adultos e idosos de municípios brasileiros de diferentes portes populacionais, além de verificar a realidade em relação à $\mathrm{AF}$ neste grupo de indivíduos, e ainda alguns fatores associados. No entanto, devemos chamar a atenção para alguns detalhes importantes tais como, as prevalências de autorrelato de DM de acordo com as regiões do Brasil. Tais resultados devem ser observados com cuidado, pois a amostra do estudo levou em consideração os diferentes portes populacionais na sua composição, o que pode determinar a não representatividade para algumas regiões do país e, portanto, prevalências subestimadas ou superestimadas. Outra questão importante deve ser observada em relação à possibilidade de causalidade reversa em algumas observações, característica dos estudos transversais. Além disso, alguns estudos demonstram que a prevalência de DM autorreferida pode ser subestimada, pois uma parcela da população não sabe que tem a doença. ${ }^{36}$

\section{CONCLUSÃO}

Os resultados apresentados mostram as prevalências de autorrelato de DM em adultos e idosos brasileiros, assim como da AF insuficiente. Observamos que embora haja um consenso sobre os benefícios da AF no controle e prevenção de diversas morbidades, entre elas o DM, o nível de AF é igual entre os indivíduos com e sem autorrelato da doença. Isso significa que mesmos aqueles indivíduos, que deveriam realizar $\mathrm{AF}$ para benefício à sua saúde, não estão praticando $\mathrm{AF}$.

\section{Contribuição dos autores:}

- Thamires Lorenzet Cunha Seus: Participou de todas as fases do artigo desde elaboração do projeto até a redação final do artigo

- Fernando Vinholes Siqueira: Participou da coleta de dados, formulação do projeto, desenvolvimento do tema, das análises de dados e da redação do artigo

- Luiz Augusto Facchini: Revisor geral do artigo compreendendo análise e redação

- Denise Silva da Silveira: Participou da coleta de dados, formulação do projeto, desenvolvimento do tema, das análises de dados e da redação do artigo

- Elaine Tomasi: Revisora do artigo e da análise.

- Elaine Thumé: Participou da redação do artigo. 
- Suele Manjourany Silva: Participou da busca bibliográfica e da redação do artigo.

- Alitéia Dilelio: Participou da revisão de bibliografia e redação do artigo.

- Roberto Xavier Piccini. Revisor do artigo em todas as suas etapas.

Todos os autores Participaram de todas as fases do Projeto AQAURES desde a formulação do projeto Inicial, desenvolvimento dos desfechos em saúde que seriam estudados, confecção dos instrumentos de pesquisa, trabalho de campo e coleta de dados, desenvolvimento da tecnologia de pesquisa com PDA.

\section{Financiamento/Agradecimento}

Ministério da Saúde do Brasil

\section{REFERÊNCIAS}

1. World Health Organization. Diabetes: diabetes facts. Fact sheet 2011 Jan No 312.

2. Schimidt MI, Duncan BB, Silva GA, et al. Chronic non-communicable diseases in Brazil: burden and current challenges. Lancet, 2011 may

3. Portal da Saúde. Disponível em: http://portal.saude.gov.br/portal/aplicacoes/noticias/default. cfm?pg=dspDetalheNoticia\&id_area=1529\&CO_NOTICIA=13914. Acessado em: Jul, 2012

4. Diretrizes da Sociedade Brasileira de Diabetes. Atualização Brasileira sobre Diabetes. (AC Farmacêutica) 2009

5. Knowler WC, Barrett-Connor E, Fowler SE, et al: Reduction in the incidence of type 2 diabetes with lifestyle intervention or metformin. N Engl J Med. 2002 346:393-403

6. The Diabetes Prevention Program (DPP) Research Group, Diabetes Care 25:2165-2171, 2002

7. Florindo AA, et al. Practice of physical activities and associated factors in adults, Brazil, 2006. Rev Saúde Publica. 2009: 43 Supp1 2:65-73.

8. Portal da Saúde. Disponível em: http://portalsaude.saude.gov.br/portalsaude/arquivos/ pdf/2012/Mai/09/Vigitel_2011_diabetes_final.pdf, Acessado em:Jul, 2012

9. Brasil. Vigitel Brasil 2008: Vigilância de Fatores de Risco e Proteção Para Doenças Crônicas por Inquérito Telefônico. Ministério da Saúde. Brasília, 2009.

10. Da Costa JS, Olinto MT, Assunção MC, Gigante DP, Macedo S, Menezes AM. Prevalence of diabetes in Southern Brazil: a population-based study. Rev Saude Publica 2006;40(3):542-5

11. Goldenberg P, Franco LJ, Pagliaro H, Silva R, Santos CA. Self-reported diabetes in the city of Sao Paulo: prevalence and inequality. Cad Saude Publica 1996;12(1):37-45.

12. Szwarcwald CL, Damacena GN. Complex sampling approaches in population surveys: planning and implications for the statistical analyses. Rev Bras de Epidemiol, 2008: 11(Supl. 1):38-45.

13. IBGE Instituto Brasileiro de Geografia e Estatística. Pesquisa Nacional por Amostras de Domicílios: acesso e utilização de serviços de saúde 2003. Brasília: Instituto Brasileiro de Geografia e Estatística; 2005.

14. Silva NN, Pedroso GC, Puccini RF, et al. Desigualdades sociais e uso de serviços de saúde: evidências de análise estratificada. Rev Saúde Pública, 2000: 34(1): 44-9.

15. Instituto Brasileiro de Geografia e Estatística. Indicadores populacionais. Rio de Janeiro: IBGE; 2000.

16. Siqueira FV, Facchini LA, Hallal PC. The burden of fractures in Brazil: A population- based study. Bone, 2005: 37(2) 261-6.

17. Meisinger C, Wildner M, Doring A, et al. Validity and realiability of problam recall of fractures. SOZ, Praeventivmed, 2000: 45:203-7.

18. Craig CL, et al. International physical activity questionnaire: 12 -country reliability and validity. Med Sci Sports Exerc, 2003: 35: 1381-95.

19. USDHHS. Physical Activity Guidelines for Americans. U.S.D.o.H.a. H. Services. 2008 Washington D.C

20. Barros AJ, Hirakata VN. Alternatives for logistic regression in cross-sectional studies: an empirical comparison of models that directly estimate the prevalence ratio. Biomedical Research Methodology, 2003: 3:21 
21. Freitas LRS; Garcia LP. Evolução da prevalência do diabetes e deste associado à hipertensão arterial no Brasil: análise da Pesquisa Nacional por Amostra de Domicílios, 1998, 2003 e 2008. Epidemiologia e Serviços de Saúde, Brasília, 2012 jan-mar 21(1):7-19

22. Francisco PMSB, et al. Diabetes auto-referido em idosos: Prevalência, fatores associados e práticas de controle. Cad Saúde Pública, Rio de Janeiro 2010 jan: 26(1):175-184

23. Bastos GAN, et al. Uso de serviços ambulatoriais nos últimos 15 anos: comparação de dois estudos de base populacional. Rev Bras Epidemiol 2011 14(4): 620-32

24. Bastos GAN, et al. Utilization of medical services in the public health system in the Southern Brazil. Rev Saúde Pública 2011:45(3)

25. Barros MBA, Francisco PMSB, Zanchetta LM, et al. Tendências das desigualdades sociais e demográficas na prevalência de doenças crônicas no Brasil, PNAD: 2003- 2008. Ciência \& Saúde Coletiva 2011; 16(9):3755-3768

26. Goodarz D, et al. National, regional, and global trends in fasting plasma glucose and diabetes prevalence since 1980: systematic analysis of health examination surveys and epidemiological studies with 370 country-years and 2.7 million participants. The Lancet, 2011 jul vol 378

27. Hypertension in Diabetes Study Group -HDS. Prevalence of hypertension in newly presenting type 2 diabetic patients and association with risc factors for cardiovascular and diabetic complications. J Hypertension, London, 1993 v.11, n.3, p.309-17

28. Centers for Disease Control and Prevention. National Diabetes Fact Sheet: National Estimates and General Information on Diabetes and Prediabetes in the United States, 2011. Atlanta, GA, U.S. Department of Health and Human Services, Centers for Disease Control and Prevention, 2011

29. Duarte CK; et al. Nível de atividade física e exercício físico em pacientes com diabetes mellitus. Rev Assoc Med Bras 2012: 58(2):215-221

30. Modeneze DM, Vilarta R, Maciel ES, et al. Nível de atividade física de portadores de diabetes mellitus tipo 2. Medicina (Ribeirão Preto) 2012: 45(1):78-86

31. Siqueira FV, Facchini LA, Silveira DS, et al. Leisure-Time Physical Activity Among Adult and Elderly Individuals in Brazil: A Countrywide Analysis. J Phys Act and Health, 2011: 8, $891-897$

32. Azevedo MR, Araújo CL, Reichert FF, et al. Gender differences in leisure-time physical activity. International J Public Health 2007. 8-15

33. Hallal PC, Dumith SC, Bastos JP, et al. Evolução da pesquisa epidemiológica em atividade física no Brasil: revisão sistemática. Rev Saúde Pública 2007 : 41(3):453-60

34. Hallal PC, Andersen LB, Bull FC, Guthold R, et al. Global physical activity levels: surveillance progress, pitfalls, and prospects. Physical Activity Series Working Group. Lancet 2012 jul

35. Siqueira FV, Nahas MV, Facchini LA, et al. Aconselhamento para a prática de atividade física como estratégia de educação à saúde. Cad Saúde Pública, Rio de Janeiro, 2009 jan 25(1):203-213

36. Malerbi DA, Franco LJ. Multicenter study of the prevalence of diabetes mellitus and impaired glucose tolerance in the urban Brazilian population aged 30-69 yr. The Brazilian Cooperative Group on the Study of Diabetes Prevalence. Diabetes Care. 1992: 15(11):1509-16.

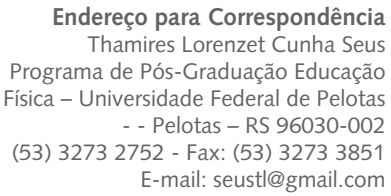

